\title{
Factors Associated with Time to Virological Failure among HIV Patients on First Line Antiretroviral at Kenyatta National Hospital Comprehensive Care Centre, Nairobi Kenya
}

\author{
Mutabari David ${ }^{1 *}$, Hillary Kipruto ${ }^{2}$, Peter Muiruri ${ }^{3}$ and Ann Wang'ombe \\ ${ }^{1}$ Project Clinician, Kenyatta National Hospital, University of Nairobi, Kenya \\ ${ }^{2}$ Senior Statistician, World Health Organization, Kenya \\ ${ }^{3}$ Project Director COEHM, Kenyatta National Hospital, Kenya \\ ${ }^{4}$ Senior Lecturer, School of Mathematics, University of Nairobi, Kenya
}

\begin{abstract}
Background: Kenya has recorded a high burden of HIV with 1.5 million HIV + persons by 2015. With the adoption of 2016 ART guideline in Kenya all PLHIV now qualify for antiretroviral (ART) irrespective of WHO clinical stage, cd4 count, age gender, pregnancy status, co-infected status thus increasing the number of patients likely to develop resistance. Once a patient develops resistance to first line, they are put on second line ART regimen which is more expensive and less tolerable. This study seeks to establish the duration of time patients put on first line ART take before developing virological failure and factors influencing it as a step towards guiding the management in adequate planning and budget allocation for management of these patients.
\end{abstract}

Study objective: To assess virological failure among HIV patients on first line ART at Kenyatta National Referral Hospital Comprehensive Care Centre between the years 2009-2016.

Methods: This was a retrospective cohort study conducted at Kenyatta National Referral Hospital Comprehensive Care Centre (KNH-CCC) between 2009 and 2016 with 3 years accrual and 6 years observation periods. The main exposure was poor adherence to first line ART treatment. HIV infected patients were enrolled and initiated into first line ART at the center between 2009 and 2010. A total of 1470 patients were selected through simple random sampling from the list of these patients in CCC database with complete follow-up records.

Data collection and analysis: A structured data abstraction tool was used for extracting sociodemographic, clinical and virological data from medical records data of the selected patients. The data ware entered and stored in Microsoft Access 2013. Data cleaning, coding and analysis was done using STATA version 13 SE. The main outcome was the virological failure time. The Kaplan-Meier estimator was used to estimate the survival function. Log-rank tests were used to compare the survival functions between patients based on adherence to treatment and first line HAART combinations. Log-rank statistic and corresponding $p$-value was reported.

Results: Generally patients sampled comprised of age 2 months to 72 years where $75 \%$ of the patients were below age $42.10 \%$ of the patients were pediatric while adults composed of $90 \%$ having female patient being almost double of the men on follow up. At enrolment clients classified in WHO ( 3 and 4 ) were $42.2 \%$ and $99 \%$ of patients were reported to have been screened for TB, $13 \%$ of patients on follow up had NCD were $64 \%$ of patients were having EFV based regimen and $35.8 \%$ had NVP based regimen. Poor adherence was reported by $34.8 \%$ of patients and $75 \%$ of patients had CD4 of less than $600 \mathrm{cell} / \mathrm{ml}$, with $19.7 \%$ of patient reported being obese and $11 \%$ were underweight. Kaplan Meier estimates indicate that $50 \%$ of adult patient failed by 36 months after ART initiation for adult's patients and for pediatric would fail by 42 months after being started on ART. Log rank tests showed that the failure rate differed significantly with relation to age, adherence and regiment type. Adults had $61 \%$ risk of virological failure at next time compared to pediatric, patient started on NVP had $18 \%$ reduced risk of virological failure compared to ones started on EFV based. Good adherence reduced chance of virological failure by $26 \%$ by the next time.

Conclusion: Patients being started on first line ART at KNH-CCC their failure time depended on age, adherence and type of regimen (EFV or NVP) where in general pediatric had longer period on first line compared to adults patients.

Copyright: (c) 2018 David M, et al. This is an open-access article distributed under the terms of the Creative Commons Attribution License, which permits unrestricted use, distribution, and reproduction in any medium, provided the original author and source are credited. 
Citation: David M, Kipruto H, Muiruri P, et al. (2018) Factors Associated with Time to Virological Failure among HIV Patients on First Line Antiretroviral at Kenyatta National Hospital Comprehensive Care Centre, Nairobi Kenya. Clin J HIV AIDS 2(1):28-35

\section{Introduction}

\section{Social value}

HIV is a global pandemic problem with no cure and HIV positive persons have to be on medication for the rest of their lives. However, without medical care, HIV still leads to AIDS and early death [1].

ARV treatment leads to longer and quality life of the HIV patients which at the end results to reduced onward transmission of the virus. Ministries of health worldwide have scaled up HIV care and treatment services and actively promoted prevention services. Many lives have so far been saved with close to 10 million adults and children being put on ART [2].

WHO advocates for public health approach towards HIV/ AIDS care and treatment and has recommended that all countries identify first line, second line and third line/salvage as treatment options for those who are infected. Treatment and other health-related support and services also need to be provided to people living with HIV is on a daily basis [3]. The annual number of new HIV infections has been steadily declining since the late 1990s and there are fewer AIDSrelated deaths due to the significant scale up of antiretroviral therapy over the past few years [4].

Within sub-Saharan region the number of new HIV infection has fallen but the prevalence is extremely high in some countries and most of them rely on donor funds to finance their HIV response [1].

Kenya is the fourth largest HIV epidemic country in the world where 1.5 million in 2015 were infected and of which $59 \%$ adults were on ART treatment. The Kenya national strategic plan defines the implementation framework of the acceleration of HIV care and treatment; thereby contribute to achieving the 90:90:90 targets set for 2019 in Kenya AIDS Strategic Framework for 2014/15-2018/2019. It will also provide guidance on high impact strategies to achieve set targets based on the county HIV burden and context [5].

The ultimate goal of HIV treatment programs is for the people living with HIV to be virally suppressed. This involves prompt diagnosis, early ART initiation and ensuring adherence of medication for the people who have been put on medication, which if not done, leads to viral mutations resulting to treatment failure among the patients.

\section{Scientific value}

The emergence of virological failure to ART drugs is an unavoidable outcome of expanding ART and the longer duration of exposure [6]. ART should be potent enough to suppress HIV viremia to undetectable levels, as measured by the most sensitive assay available, and which is durable in its virologic effect [7]. Clients who had failed on first-line drug have a higher percentage of more than $46 \%$ failing again for second-line drugs; this contributes to the higher number of side effects with greater likelihood of drug resistance and treatment fatigue [8]. A delay if seeking for treatment failure can also lead to increase in drug toxicity that may lead to the buildup of drug resistance-associated mutations. It may also result in increased morbidity and mortality [8].
Many studies indicates that many HIV + clients who fail the second line ART in resource-limited settings require major PI resistance-associated mutations, this implies that there is failure due to reduced adherence [6] thus concentrating on first line treatment failure will help know the average time a patient take on first line ART medication before developing virological failure.

\section{Conceptual framework}

The main aim of antiretroviral treatment for HIV persons is to suppress viral load to undetectable levels thus enabling them to live a long and high quality life free of opportunist infections. Ideally a patient should have undetectable viral load from six months of starting ART or having been changed from one combination of ART to another in the event of a validated virological treatment failure.

With the increasing number of patients being put on treatment at Kenyatta National Referral Hospital Comprehensive care Center ( $\mathrm{KNH}-\mathrm{CCC}$ ) mainly due to the new $\mathrm{WHO} /$ National guideline of test and treat guidelines, the number of clients who fail on first line has been increasing proportionally over time. No studies have establish how long patients remain on ART remain on treatment before drugs lose their potency to fight the virus given or what are the factors contributing to some patients failing earlier than others. With this, there are a number of clients having detectable viral load after short period of initiation of first line ART making them candidate for second line treatment. These treatment are more expensive, and the combinations of the drugs needed have not been availed readily to patients (public hospitals) making adherence more complex compared to first line ART.

This study sought to find out the time taken for HIV patients to fail i.e. (based on detectable viral load after six months of initiating treatment) on first line with the associated factors contributing to this failure at $\mathrm{KNH}-\mathrm{CCC}$. The findings can be used to address challenges arising with treatment failure and help have more specific targeted address to challenges facing clients being started on ART or still on follow up. Additionally findings will form a basis for future research to be conducted at the clinic or elsewhere and evidence for policy makers in line with HIV patient management.

\section{Aims and objectives}

The broad objective was to assess virological failure time and the factors associated among HIV patients on first line antiretroviral at KNH-CCC between 2009 and 2016. Specific

*Corresponding author: Mutabari David, MSC, Project Clinician, Kenyatta National Hospital, University of Nairobi, Box 20750-00202, Kenya, Tel: 254722441165

Accepted: November 21, 2018

Published online: November 23, 2018

Citation: David M, Kipruto H, Muiruri P, et al. (2018) Factors Associated with Time to Virological Failure among HIV Patients on First Line Antiretroviral at Kenyatta National Hospital Comprehensive Care Centre, Nairobi Kenya. Clin J HIV AIDS 2(1):28-35 
Citation: David M, Kipruto H, Muiruri P, et al. (2018) Factors Associated with Time to Virological Failure among HIV Patients on First Line Antiretroviral at Kenyatta National Hospital Comprehensive Care Centre, Nairobi Kenya. Clin J HIV AIDS 2(1):28-35

objectives were to determine time taken for patients to develop virological failure (VF) among patients enrolling on first line ART at KNH-CCC, to compare time to (VF) among HIV patients initiated on different first line ART combinations at KNH CCC and to evaluate factors associated with (VF) among patients on first line.

\section{Research Methods and Design}

\section{Study design}

A retrospective cohort study of HIV patients enrolled on first line ART at KNH-CCC between 1st January, 2009 and 31st December, 2013 was identified and medical records were abstracted to determine clinical data, pharmacy data and laboratory investigation including VF through December 31, 2016.

\section{Study site}

The study was conducted at Kenyatta National Hospital at the Comprehensive Care Center clinic in upper hill Nairobi which is an ongoing HIV clinic supported by CDC and working with collaboration of University of Nairobi running from Monday to Friday serving all patients who can follow set national guideline for HIV clinic services.

\section{Study population}

The study included a representative sample of HIV patients initiated into first line ART between January 2009 to December 2011 which served as the recruitment period and were being followed-up at $\mathrm{KNH}-\mathrm{CCC}$ up to December 2016. Each client was followed up for six years from the time of initiation. The clinic had a total of 9915 active patients on follow up at the end of 2016 with 9063 being classified as adults ( $\geq 20$ years), 362 as adolescents (15-19 years) and
693 as pediatrics (0-14 years). On first line there were 8262 adults, 284 adolescents and 662 children whereas second line comprised of 780 adults, 74 adolescents and 28 children. The few remaining (24 patients) were on third line.

\section{Sampling method}

A sampling frame of eligible patients who initiated ART at KNH-CCC between January 1, 2009 and December 31, 2011 was identified using the hospitals electronic medical records system (IQ-CARE). Patients were grouped into strata based on age group ( $<15$ years $\geq 15$ ) and six different types of first line ART used at the clinic.

Simple random sampling with equal allocation was used to select 1331 adults and 141 children with a total sample of 1472 .

\section{Data collection}

The IQ-CARE data extraction tool was used to download available visit data for each selected patient stored in MS Access version 2013.

\section{Data Management and Analysis}

Data were imported to STATA version 13SE for cleaning and coding. Exploratory data analysis was then done to summarize the data. Histograms were plotted to show the distribution of quantitative variables and measures of central tendency (mean/median) and dispersion (standard deviation/ inter-quartile range) reported in tables. For categorical variables bar/pie charts we plotted to show the distribution and frequencies and proportions reported in tables. The Kaplan-Meier method was used to estimate survival functions.

Log-rank tests were used to compare the survival functions based on the different first line ART combinations. Log-rank statistic and corresponding $\mathrm{p}$-value were reported.

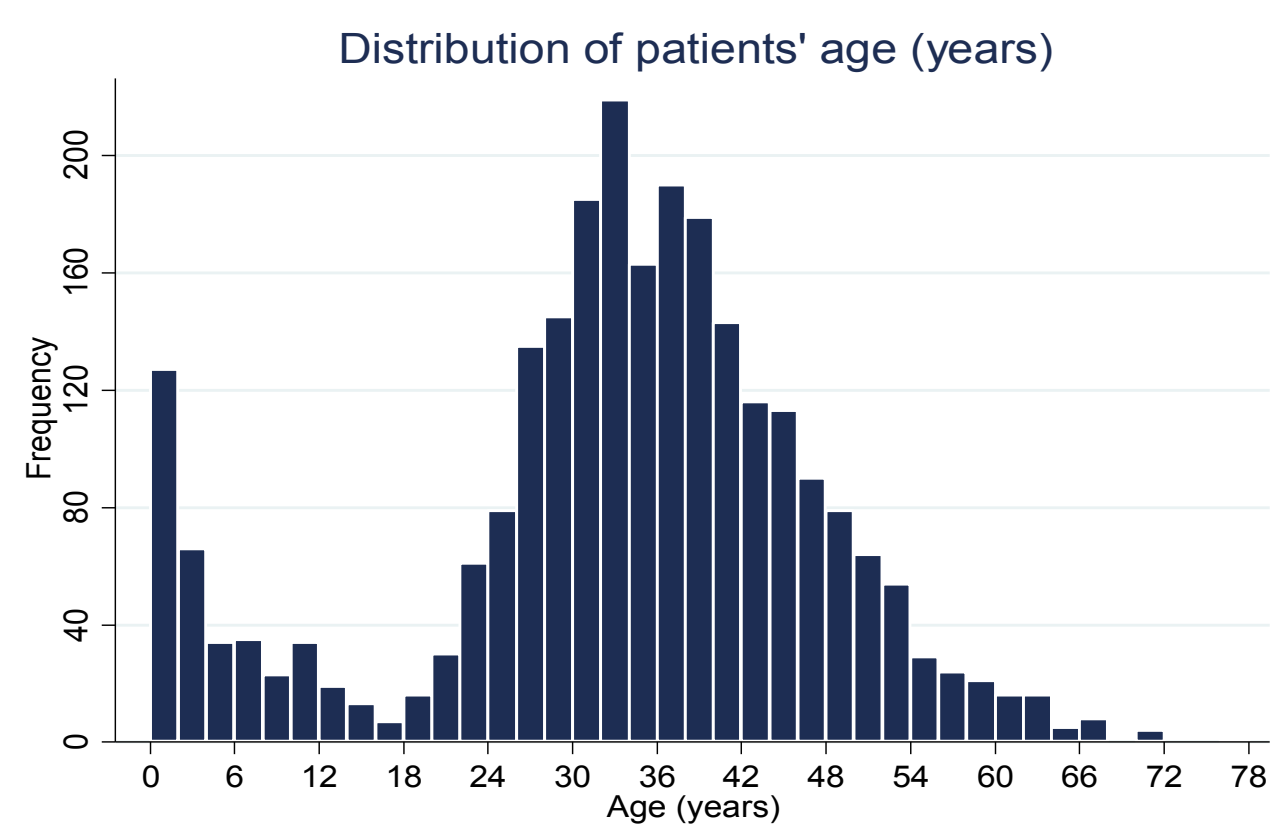

Figure 1: Distribution of the patients' age (years).

Histogram shows distribution of the 1472 patients sampled for the study from age of 2-months-old to age of 72 -years-old indicating that three quarter of patients in the study were less than 42 years of age. 
Citation: David M, Kipruto H, Muiruri P, et al. (2018) Factors Associated with Time to Virological Failure among HIV Patients on First Line Antiretroviral at Kenyatta National Hospital Comprehensive Care Centre, Nairobi Kenya. Clin J HIV AIDS 2(1):28-35

Cox proportional hazards model was used to evaluate prognostic factors for virological failure among patients on first line ART and the assumption was not violated thus frailty model was adopted to estimate the hazard ratios. The assumptions for proportional hazard were evaluated. Hazard ratios and corresponding confidence intervals were reported.

\section{Ethical Considerations}

Ethical approval was sought from the University of Nairobi/ Kenyatta National Hospital ethics board where additional permission from the Kenyatta national hospital management health system and head of unit at Kenyatta national hospital comprehensive care center to use the electronic medical records servers was granted.

\section{Results}

\section{Profile of the HIV patients at KNH-CCC}

Data was collected from a total of 1,472 HIV positive patients

Table 1: Demographic characteristics of the patients.

\begin{tabular}{|l|l|l|}
\hline Variable & Category & Frequency \\
\hline Gender & Female & $892(60.6)$ \\
\cline { 2 - 3 } Age group & Male & $580(39.4)$ \\
\hline \multirow{2}{*}{ BMI } & $<15$ years & $141(9.6)$ \\
\cline { 2 - 3 } & $\geq 15$ years & $1331(90.4)$ \\
\hline & Underweight & $166(11.4)$ \\
\cline { 2 - 3 } & Normal weight & $573(39.3)$ \\
\cline { 2 - 3 } & Overweight & $432(29.6)$ \\
\hline & Obese & $287(19.7)$ \\
\hline
\end{tabular}

Demographic characteristic of the 1472 patient sampled for the study which shows there are almost twice females on follow up as men and the larger group on follow up are above 15 years of age.

Table 2: Clinical profile of the patients at baseline and treatment history.

\begin{tabular}{|l|l|l|}
\hline Variable & Category & Frequency \\
\hline WHO stage & Stage 1 & $398(33.0)$ \\
\hline & Stage 2 & $299(24.8)$ \\
\hline & Stage 3 & $384(31.8)$ \\
\hline Stage 4 & $125(10.4)$ \\
\hline TB screening results & No signs/symptoms & $1398(99.0)$ \\
\hline & On treatment & $6(0.4)$ \\
\hline Any non-communicable disease & Suspected TB & $8(0.6)$ \\
\hline Regimen type & Yes & $1268(86.1)$ \\
\hline Adherence & EFV & $204(13.9)$ \\
\hline NVP & $945(64.2)$ \\
\hline CTX dapsone & Good & $527(35.8)$ \\
\hline Missed doses & Poor & $959(65.2)$ \\
\hline Delays in taking medication & Yes & $513(34.8)$ \\
\hline & No & $1472(100)$ \\
\hline & Nes & $1059(98.5)$ \\
\hline Yes & $16(1.5)$ \\
\hline & & $1054(98.9)$ \\
\hline
\end{tabular}

Presentation of the clinical profile of the patient enrolled for the study at baseline level which most significant thing was screening of TB among patient being enrolled up to $99 \%$. who were initiated into antiretroviral therapy between January, 2009 and December 2010 at KNH comprehensive care clinic (Figure 1).

The age distribution was bimodal as shown in the graphs; with peaks at 1 year and 33 years. The patients' age ranged from months to 2 months to 72 years. Three quarters of the patients in this study were younger than 41.9 years.

Demographic characteristics of the 1472 patients enrolled for the study shows distributions in terms of age groups, adult patients ( $\geq 15$ years were) the most predominant constituting $90 \%$ of the population. The rest were pediatric patients $(<15$ years of age) (Table 1).

There were more female patients (almost twice the number) than male patients. Only $39.3 \%$ of the patients had a normal BMI at the start of ART.

For the clinical profile of the patient sampled for the study at baseline shows $67.0 \%$ of the patients started treatment when the HIV infection had progressed beyond stage 1. Almost all (99\%) the patients that had undergone TB screening did not present any signs \& symptoms of TB infection. $13.9 \%$ of the patients were suffering from at least one non-communicable disease e.g. hypertension, diabetes etc. (Table 2).

More than half of the patients (64.2\%) were started on EFV based ART regimens. The rest were on NVP based regimens. Using the WHO drug adherence assessment guidelines, $35.8 \%$ of the patients were considered as a having poor drug adherence. Almost all the patients did not miss their doses (98.5\%) or delay in taking medication (98.9\%). CD4 count distribution for the patient sampled shows a right skewed. CD4 count ranged from 2 cells $/ \mathrm{ml}$ to 2507 cells $/ \mathrm{ml}$ with three quarters having less than 590 cells/ml (Figure 2).

\section{Estimation of virological failure time}

Kaplan Meier method was used to estimate the virological failure function in HIV patients receiving HIV treatment at $\mathrm{KNH}-\mathrm{CCC}$. Quarterly time interval was used in the model (Figure 3).

Starting with 1472 patients at risk of virological failure, $50 \%$ would fail before time 12 , which represents 36 months or three years after initiation of ART. For the first 18 months (between time 0 and 6 ), the probability of virological failure was zero, after which a steady increase in the probability to fail with respect to time was observed until time 15 (45 months later). This was followed by a gradual increase until the end of the study.

\section{Estimated virological failure time of pediatric patients}

Kaplan Meier method was further applied to find the probability of a 141 pediatric patient sampled to fail virologically which was zero for the first 18 months (up to time 6 ) after which a gradual increase was observed until the end of the study (Figure 4).

Estimation of virological failure time of adult 


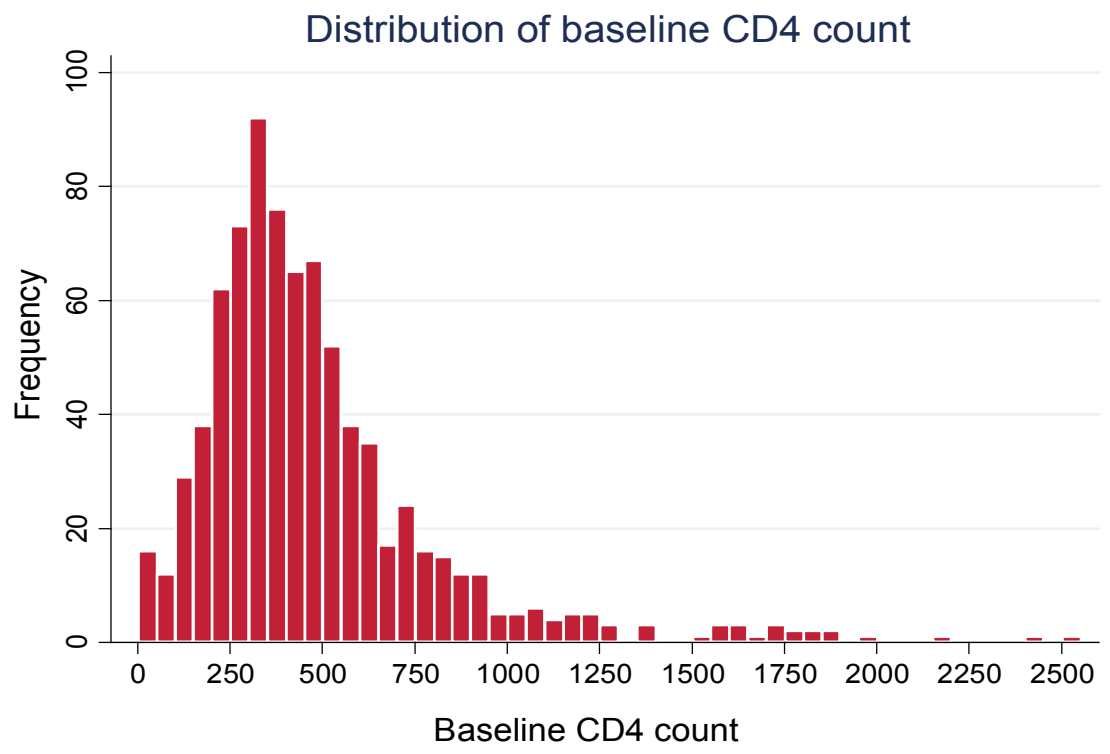

Figure 2: Distribution of the CD4 count in patients at baseline.

Graph showing CD4 count distribution for patient enrolled at baseline where the lowest is 2 cells to the highest being 2507 cells.

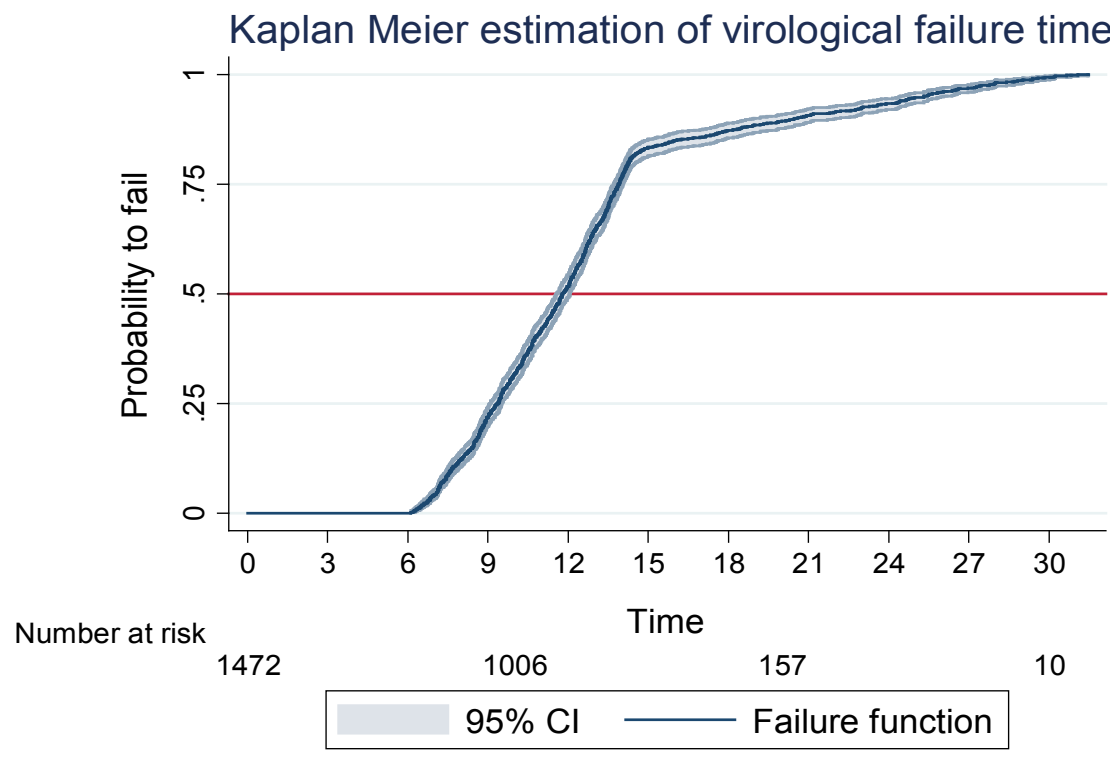

Figure 3: Kaplan Meier Estimation of virological failure function in quarterly intervals.

Kaplan Meier graph shows the virological failure function for HIV patients receiving treatment on quarterly time interval.

\section{patients}

Virological failure time for the adult patient has been (Figure 5) demonstrates that between time 0 and 6 , the probability of an adult patient to fail was zero; after time 6 up to time 15, a steady increase in the probability to fail was observed followed by a gradual increase until the end of the study. $50 \%$ of the patients on ART would fail virologically before time 12 i.e. by the $3^{\text {rd }}$ year (Figure 5).

\section{Comparison of failure functions using log rank tests}

Log rank tests was applied for the sampled patients to compare the failure functions by age group, gender, ART regimen type, adherence to treatment and stage of the disease. The test showed that the failure functions differed significantly between adult and pediatric patients $(p<0.001)$; between patients on EFV and NVR based regimens $(p=0.024)$ as well as between patients with good and poor adherence to ART $(p<0.001)$ (Table 3).

\section{Determinants of virological failure}

A Cox Proportional Hazards regression model was fit to evaluate the adjusted effect of the patient's baseline characteristics on the risk of virological failure at the next time. Only age group, ART regimen type and adherence to ART were found to have a significant effect on the risk of virological failure at the next time (Table 4). 
Citation: David M, Kipruto H, Muiruri P, et al. (2018) Factors Associated with Time to Virological Failure among HIV Patients on First Line Antiretroviral at Kenyatta National Hospital Comprehensive Care Centre, Nairobi Kenya. Clin J HIV AIDS 2(1):28-35

Kaplan Meier estimation of virological failure time in paediatric patients

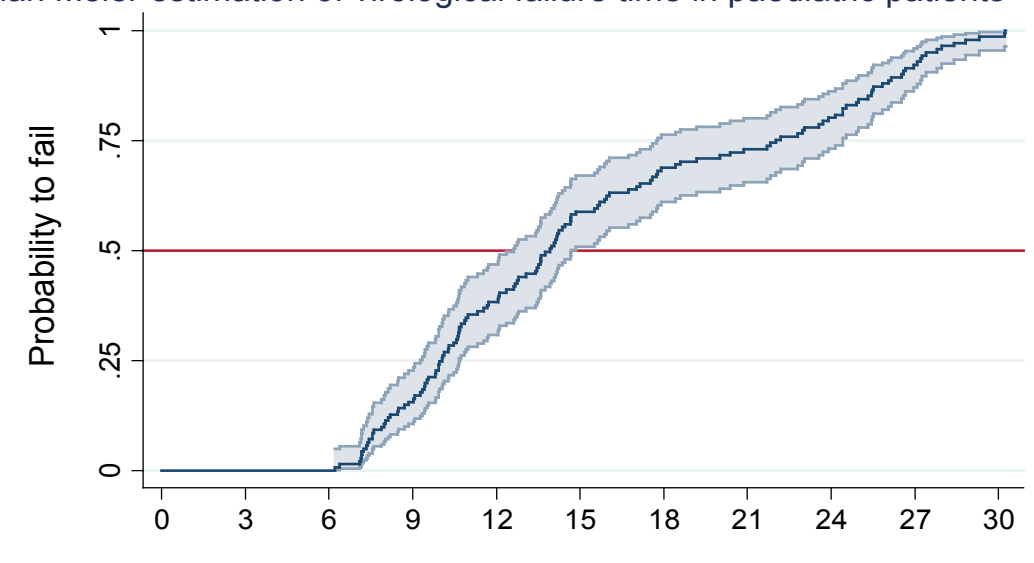

Number at risk

141

\begin{tabular}{|cc|}
106 & 41 \\
\hline $95 \% \mathrm{Cl}$ & Failure function \\
\hline
\end{tabular}

Figure 4: Estimated virological failure time of pediatric patients.

Shows graph of virological failure time among pediatric patients enrolled for the study at the clinic. Starting with 141 pediatric patients at risk of virological failure, $50 \%$ would die before time 14 (before the $42^{\text {nd }}$ month) from the time of ART initiation.

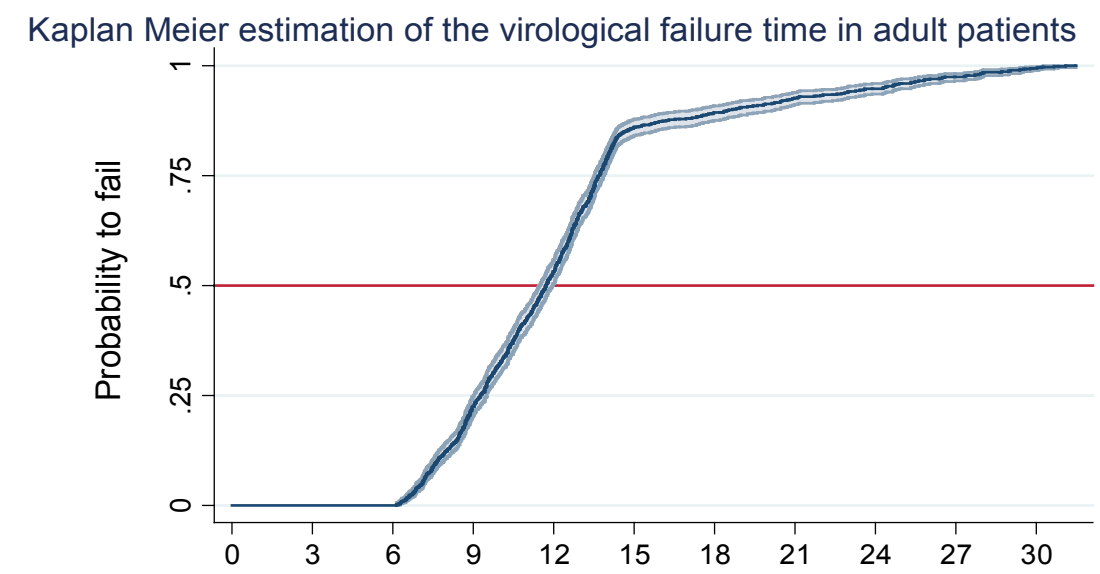

Number at risk

Time spent

1331

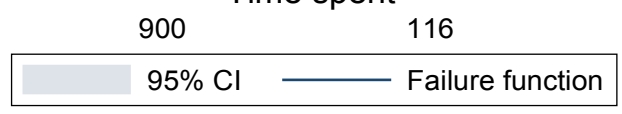

Figure 5: Kaplan Meier estimation of virological failure time of adult patients.

Shows graph presentation of the virological failure time among the adult patients enrolled for the study where $50 \%$ of the patients would fail by the third year of follow up.

Table 3: Comparison of virological failure functions.

\begin{tabular}{|l|l|l|l|}
\hline Variable able & $\begin{array}{l}\text { Observed } \\
\text { events }\end{array}$ & $\begin{array}{l}\text { Log-rank } \\
\text { test chi }\end{array}$ & P-value \\
\hline Age group & \multicolumn{3}{|l|}{} \\
\hline Paediatric (< 15 years) & 141 & 32.59 & $<0.001^{*}$ \\
\hline Adult ( $\mathbf{1 5}$ years) & 1331 & & \\
\hline Gender & & & \\
\hline Female & 892 & 1.95 & 0.163 \\
\hline Male & 580 & & \\
\hline Regimen type & & & \\
\hline EFV & 945 & 5.10 & $0.024^{*}$ \\
\hline NVP & 527 & & \\
\hline
\end{tabular}

\begin{tabular}{|l|l|l|l|}
\hline HIV stage & \multicolumn{3}{l|}{} \\
\hline Stage 1 & 398 & 0.99 & 0.805 \\
\hline Stage 2 & 299 & & \\
\hline Stage 3 & 384 & & \\
\hline Stage 4 & 125 & & \\
\hline Adherence & \multicolumn{3}{|l|}{} \\
\hline Good & 959 & 43.55 & $<0.001^{*}$ \\
\hline Poor & 513 & & \\
\hline
\end{tabular}

"significant. Shows log rank test comparison of the virological failure by time age group, gender, ART regimen type, adherence to treatment and stage of the disease. 
Citation: David M, Kipruto H, Muiruri P, et al. (2018) Factors Associated with Time to Virological Failure among HIV Patients on First Line Antiretroviral at Kenyatta National Hospital Comprehensive Care Centre, Nairobi Kenya. Clin J HIV AIDS 2(1):28-35

Table 4: Cox proportional hazards regression-determinants of virological failure.

\begin{tabular}{|c|c|c|c|}
\hline Variable e & Hazard ratio & $95 \% \mathrm{Cl}(\mathrm{HR})$ & P-value \\
\hline \multicolumn{4}{|l|}{ Age group } \\
\hline Paediatric (< 15 years) & (base) & & \\
\hline Adult ( $\geq 15$ years) & 1.61 & {$[1.19 ; 2.16]$} & $0.002^{*}$ \\
\hline \multicolumn{4}{|l|}{ Gender } \\
\hline Female & (base) & & \\
\hline Male & 1.04 & {$[0.89 ; 1.21]$} & 0.609 \\
\hline \multicolumn{4}{|l|}{ Regimen type } \\
\hline EFV & (base) & & \\
\hline NVP & 0.82 & {$[0.70 ; 0.96]$} & $0.012^{*}$ \\
\hline \multicolumn{4}{|l|}{ HIV stage } \\
\hline Stage 1 & (base) & & \\
\hline Stage 2 & 0.94 & {$[0.76 ; 1.16]$} & 0.560 \\
\hline Stage 3 & 0.94 & {$[0.77 ; 1.14]$} & 0.517 \\
\hline Stage 4 & 0.92 & {$[0.70 ; 1.22]$} & 0.586 \\
\hline \multicolumn{4}{|c|}{ Any non-communicable disease? } \\
\hline No & (base) & & \\
\hline Yes & 1.08 & {$[0.87 ; 1.34]$} & 0.466 \\
\hline \multicolumn{4}{|l|}{ Adherence to ART } \\
\hline No & (base) & & \\
\hline Yes & 0.74 & {$[0.62 ; 0.87]$} & $<0.001^{*}$ \\
\hline \multicolumn{4}{|l|}{ Baseline BMI } \\
\hline Normal & (base) & & \\
\hline Obese & 0.95 & {$[0.76 ; 1.19]$} & 0.671 \\
\hline Overweight & 1.05 & {$[0.86 ; 1.28]$} & 0.628 \\
\hline Underweight & 1.09 & {$[0.82 ; 1.46]$} & 0.540 \\
\hline
\end{tabular}

*significant. Shows Cox Proportional Hazards regression model evaluating the adjusted effect of the patient's baseline characteristics on the risk of virological failure at the next time.

An adult patient has $61 \%$ increased risk of virological failure at the next time (after 36 months of follow up) compared to a pediatric patient, adjusting for the effect of the other covariates in the model. Controlling for the effect of other covariates, patients started on NVP regimens had $18 \%$ reduced risk of virological failure at the next time (after 36 months of follow up) compared to patients started on EFV based regimens.

A patient who adheres to treatment has a $26 \%$ reduced risk of virological failure at the next time (after 36 months or 45 moths of follow up) than a patient who does not adhere to treatment, adjusting for the effect of the other covariates.

\section{Discussion}

The use of HIV treatment not only improves the health of people living with HIV, but is also a highly effective strategy to prevent HIV transmission. This is because HIV treatment can reduce the amount of virus (viral load) in the blood and other bodily fluids (such as semen and vaginal and rectal fluids) to undetectable levels. To achieve and maintain viral suppression, people living with HIV need to take their ART treatment as prescribed. In addition to taking ART medications, regular medical visits are important to monitor viral load [9], to make sure it stays undetectable, and to receive other medical support.

The aim of this study was to find out what factors influence the time a client registered at Kenyatta National Hospital Comprehensive Care $\mathrm{C}$ enter takes to start developing virological failure; a key reason for change of treatment to second line regimen. The study evaluated the association of the baseline characteristics (age, regimen type, adherence, gender, HIV stage, presence of non-communicable disease(s), and $\mathrm{BMI}$ ) of the patients with the risk of virological failure using the Cox proportional hazards model. The age of the patient, first line regimen type and adherence to treatment were significantly associate with the time to failure. The median time to failure in the overall population was estimated at 36 months, which is comparable to the median time to failure reported in a study conducted at AMPATH program in Western Kenya [10].

Though their criteria for categorizing age was different from this study, studies by [9] in North West Ethiopia, [11] Hassan, et al. in Coastal Kenya, and [12] in Nyanza region, Kenya have also reported age of the patient as a significant risk factor for virological failure. In this study adult patients were found to have a significant $61 \%$ increased risk of failing at the next time compared to children. This finding contrasts with the study findings from a study in Swaziland, where children were found to have a 2.6 times higher odd of having detectable viral load compared to adults [13]. In this study the high risk of virological failure was attributed to the limited available evidence on viral load monitoring in children giving room to poor adherence to treatment an important risk factor for failure. At $\mathrm{KNH}-\mathrm{CCC}$, however the monitoring of all patients especially pediatric patients is done actively and reminders sent out to patients missing their clinics as well as counselling to caregivers of HIV infected children. This could perhaps explain why the risk of failure among the children in this study was lower compared to adult patients.

Patients started on Nevirapine regimen had an $18 \%$ reduced risk of failure at the next time compared to patients on Efavirenz. The risk estimate in this study was comparable to the odd reported by the AMPATH study [10] however this association between regimen and failure was not statistically significant as the case in our study. Previous studies have suggested that Nevirapine-based ART is marginally less efficacious compared Efavirenz based regimen but association tests found no significant difference in the risk of virological failure [14].

Adherence to antiretroviral therapy (ART) is of critical importance because even minor deviations from the prescribed regimen can result in viral resistance [15]. The study found that those patients who adhere to treatment had $26 \%$ reduced risk of virological failure in this clinic. With $34 \%$ of patients found to have poor adherence to treatment the clinicians need to prioritize on coming up with strategies to improve adherence to treatment to reduce incidences of drug resistance given the current test and treat policy where an upsurge of patients in care is likely to be experienced.

Clinically $67 \%$ of patients started on treatment at the facility, were in WHO stage two and above signifying how rarely people check their HIV status and start treatment. The clinical stage of the HIV infection was not associated with the 
Citation: David M, Kipruto H, Muiruri P, et al. (2018) Factors Associated with Time to Virological Failure among HIV Patients on First Line Antiretroviral at Kenyatta National Hospital Comprehensive Care Centre, Nairobi Kenya. Clin J HIV AIDS 2(1):28-35

risk of virological failure in this study irrespective of when they started HAART.

\section{Conclusion}

The study found age group of patient, type of regimen (Nevirapine/Efavirenz) adherence to treatment to be significantly associated with the time to virological failure. Half of the patients are likely to start failing after 36 months from the start of treatment. Pediatric patients take longer before they start failing compared to adult patients. Health care providers managing the HIV patients should be alerted on the need for ART treatment support that addresses adherence problems especially in adult patients to improve adherence consequently reducing the risk for virological failure due to resistance. There is need to conduct further studies on the association between type of regimen and risk of failure to come up with conclusive report as to whether Nevirapine based regimens are better compared to Efavirenz in sustenance of suppressed viral load.

\section{Acknowledgments}

This goes to the Kenyatta national Hospital data team who enabled me abstract the data of patient sampled for the study and my clinical team for their support as I collected data at the hospital.

\section{References}

1. (2016) Today's HIV/AIDS Epidemic. Scope T 2-5.

2. Essajee S, Kumarasamy N (2014) Commentary. Aids 28 S147-S149.

3. http://www.euro.who.int/en/publications/abstracts/hivaids treatment-and-care.-clinical-protocols-for-the-who-europeanregion

4. Getnet $Y$ (2014) Determinants of first line antiretroviral treatment failure in public hospitals of Addis Ababa, Ethiopia: Unmatched case control study. J Biol Agric Healthc 4: 1-12.
5. (2016) Health of national guidelines for HIV prevention treatment and care national aids and sti's control.

6. Meintjes G, Dunn L, Coetsee M, et al. (2015) Third-line antiretroviral therapy in Africa: Effectiveness in a Southern African retrospective cohort study. AIDS Res Ther 12: 39.

7. Sik-to LAI, Chin-peng LEE, Chung-ki LI, et al. (2011) Recommended principles of antiretroviral therapy in HIV disease.

8. Care $P$ (2016) First-line antiretroviral treatment failure and associated factors in HIV patients at the university of gondar teaching hospital, Gondar, Northwest Ethiopia. HIV AIDS (Auckl) 141-146.

9. Bayu B, Tariku A, Bulti A, et al. (2017) Determinants of virological failure among patients on highly active antiretroviral therapy in university of gondar referral hospital, Northwest Ethiopia: A case-control study. HIV AIDS (Auckl) 9: 153-159.

10. Kwobah CM, Mwangi AW, Koech JK, et al. (2012) Factors associated with first-line antiretroviral therapy failure amongst HIV-infected african patients: A case-control study. Scientific Researcher.

11. Hassan AS, Nabwera HM, Mwaringa SM, et al. (2014) HIV-1 virologic failure and acquired drug resistance among first-line antiretroviral experienced adults at a rural HIV clinic in coastal Kenya: A cross-sectional study. AIDS Res Ther 11.

12. Sang RKA, Miruka FO (2016) Factors associated with virologic failure amongst adults on antiretroviral therapy in nyanza region, Kenya. IOSR Journal of Dental and Medical Sciences (IOSR-JDMS) 15: 108-121.

13. Jobanputra K, Parker LA, Azih C, et al. (2015) Factors associated with virological failure and suppression after enhanced adherence counselling, in children, adolescents and adults on antiretroviral therapy for HIV in Swaziland. PLoS One 10: 1-12.

14. Manfredi R (2004) Impact of HIV infection and antiretroviral therapy in the older patient. Expert Rev Anti Infect Ther 2: 821824.

15. Chaiyachati KH, Ogbuoji O, Price M (2014) Interventions to improve adherence to antiretroviral therapy: A rapid systematic review. AIDS 187-204.

DOI: $10.36959 / 695 / 565$ 